\title{
Política Nacional de Atenção Básica: para onde vamos?
}

\author{
National Primary Health Care Policy: where are we headed to?
}

Ligia Giovanella (https://orcid.org/0000-0002-6522-545X) ${ }^{1}$

Cassiano Mendes Franco (http://orcid.org/0000-0003-1430-6951) ${ }^{2}$

Patty Fidelis de Almeida (https://orcid.org/0000-0003-1676-3574) ${ }^{3}$

${ }^{1}$ Escola Nacional de Saúde Pública Sérgio Arouca, Fundação Oswaldo Cruz. R. Leopoldo Bulhões 1480, Manguinhos. Rio de Janeiro RJ Brasil.

ligiagiovanella@gmail.com ${ }^{2}$ Faculdade de Medicina,

Universidade Federal do Rio

de Janeiro. Rio de Janeiro

RJ Brasil.

${ }^{3}$ Instituto de Saúde da Comunidade, Universidade Federal Fluminense. Niterói RJ Brasil.

\begin{abstract}
This paper analyzes recent policies in the field of Primary Health Care (PHC) and their possible implications for the care model in the Unified Health System (SUS). Initially, some of the concepts that influenced the models of care in the Brazilian public system are revived, and we argue that the Family Health Strategy (ESF) bases for reorienting care practices in primary care are consistent with the principles of the SUS. Below, we analyze the central elements of new federal policies for PHC. We show that changes in the PHC care model threaten the teams' multidisciplinarity, prioritize acute illness care, focus in individual care, weaken the community territorial approach and establish coverage by registration, which evidence redirection of the health policy, harming the principles of universality, integrality, and equity in the SUS.
\end{abstract}

Key Words Primary health care, Health policy, Healthcare models
Resumo $O$ artigo analisa políticas recentes no campo da Atenção Primária à Saúde (APS) e suas possíveis implicações para o modelo assistencial no Sistema Único de Saúde (SUS). Inicialmente resgatam-se algumas das concepções que influenciaram os modelos de atenção no sistema público brasileiro e argumenta-se que a Estratégia Saúde da Família (ESF) apresenta as bases para reorientação das práticas assistenciais na atenção básica coerentes com os princípios do SUS. A seguir, analisam-se elementos centrais de politicas federais recentes para a APS. Demonstra-se que as mudanças no modelo assistencial da APS com ameaças à multiprofissionalidade das equipes, prioridade ao pronto atendimento, centralidade no cuidado individual, enfraquecimento do enfoque territorial comunitário e cobertura por cadastramento, evidenciam redirecionamento da política de saúde, ferindo os princípios da universalidade, integralidade e equidade no SUS.

Palavras-chave Atenção primária à saúde, Política de saúde, Modelos de assistência à saúde 


\section{Introdução}

Ao comemorarmos os 40 anos da Declaração de Alma-Ata sobre atenção primária à saúde (APS) que inspira por décadas movimentos sociais, ativistas, profissionais e governos defensores do direito universal à saúde mundo afora, nós brasileiros nos defrontamos com retrocessos, ameaças e desmesurados novos desafios. A erosão dos direitos sociais e o cerceamento dos direitos civis e políticos promovidos pelo governo Bolsonaro fere a democracia, agrava os determinantes sociais da doença e, no setor saúde, ameaçam a universalidade, integralidade e equidade no Sistema Único de Saúde (SUS).

Este artigo analisa políticas recentes no campo da APS e discute suas implicações para o modelo de atenção. Inicialmente resgata sentidos dos modelos de atenção em disputa que informam a implementação do SUS. Defende-se que a Estratégia Saúde da Família (ESF) apresenta bases para reorientação das práticas assistenciais na atenção básica coerentes com os princípios do sistema público brasileiro. A seguir analisam-se elementos centrais de políticas federais para a APS. Argumenta-se que a direcionalidade das políticas públicas, sobretudo a partir de 2017, sinaliza mudanças e ameaças ao modelo assistencial da ESF e aos princípios da universalidade, integralidade e equidade no SUS.

\section{A ESF e a mudança do modelo de atenção à saúde}

Caracteriza-se modelo de atenção como a "lógica ou racionalidade que orienta uma dada combinação tecnológica nas práticas em saúde" ou "modos de organizar a ação e dispor os meios técnicos científicos para intervir sobre problemas e necessidades de saúde" individuais e coleti$\operatorname{vos}^{1}($ p.463). Envolve o modo como são disponibilizados os recursos (humanos e materiais), as tecnologias (materiais e não materiais), a rede de serviços, as práticas e relações entre profissionais e população, nas dimensões política, gerencial e organizativa ${ }^{1,2}$.

A criação do SUS implicou em mudanças do modelo de atenção nas diversas dimensões, especialmente na política e gerencial, contudo, não se estabeleceu em sua criação um novo modelo de APS, ainda que influenciado por propostas com distintas racionalidades e experiências localizadas nas décadas precedentes ${ }^{1}$. Rechaçava-se a vigência da medicina previdenciária, caracterizada pelo acesso restrito e estratificado à saúde, pela dicoto- mia entre assistência e prevenção e pela concepção biomédica e hospitalocêntrica do cuidado. Já na $8^{a}$ Conferência Nacional de Saúde, além da prerrogativa da universalidade, integralidade e equidade, defendia-se a APS e a promoção da saúde. Todavia, é a partir da década de 1990 que um modelo para reorientar a prática assistencial na APS, o Programa Saúde da Família (PSF) ocuparia o que Viana e Dal Poz denominaram como "vazio programático" desde a criação do SUS - ainda que originalmente dirigido a grupos da população sem acesso e com escopo seletivo de ações ${ }^{2,4}$.

Paim $^{1}$ subordina a iniciativa do PSF e de seu antecessor, o Programa Agentes Comunitários de Saúde (PACS), em seus primórdios, ao modelo que denomina de "sanitarista", caracterizado por programas verticais e hierarquizados, voltados ao controle de determinadas doenças. Não obstante, entende que o PSF foi redefinido progressivamente como uma estratégia de mudança dos modelos de atenção hegemônicos ${ }^{1}$. A Estratégia Saúde da Família (ESF) caracteriza-se como modelo alternativo que busca aliar a prática do cuidado individual à abordagem populacional na perspectiva da vigilância em saúde, integrar vigilância epidemiológica e sanitária, territorialização/distritalização, atenção clínica e políticas intersetoriais, ações programáticas e reorganização do atendimento à demanda espontânea com acolhimento centrado no usuário, consolidando diretrizes e princípios do SUS como universalidade, integralidade e equidade ${ }^{2,4}$.

O lançamento e distribuição a todas as equipes de saúde no país do livro de Barbara Starfield $^{5}$ Atenção Primária: equilíbrio entre necessidades de saúde, serviços e tecnologia, em 2002, na Escola Nacional de Saúde Pública Sergio Arouca/ Fiocruz, difundiu uma certa concepção e organização da APS a partir de atributos essenciais (primeiro contato, abrangência/integralidade, longitudinalidade/vínculo e coordenação) e derivados (orientação familiar, orientação comunitária e competência cultural), incorporados, posteriormente à Política Nacional de Atenção Básica em $2006^{4,6}$. Os atributos da $\mathrm{APS}^{5}$, além de direcionarem políticas e práticas, incorporam princípios presentes na atuação e formação em Medicina de Família e Comunidade (MFC). A partir dos anos 2000, cargos centrais na condução da política de atenção básica no Ministério da Saúde (MS), em municípios e estados com projeção nacional foram ocupados por gestores com formação em MFC, bem como foram implantadas políticas para formação e provimento incentivando a formação na especialidade. 


\section{Influência da MFC nos modelos assistenciais na APS}

A MFC no Brasil tem suas origens na década de 1970, quando foram criados programas de Medicina Comunitária organizados, em grande parte, pelos departamentos de Medicina Preventiva, como iniciativas de integração docente-assistencial ${ }^{7}$. Embora representassem reação à especialização da prática médica e reivindicação por reformas sociais mais amplas, os programas contavam também com financiamento de agências internacionais como a Fundação Kellog e Ford com concepções focalizadas de saúde e elementos da prática médica liberal, o que resultou em críticas do movimento da Saúde Coletiva ${ }^{7,8}$. Donnangelo $^{9}$ argumentava que a proposta da Medicina Comunitária não mudava a forma de conceber a prática. Ainda que voltada para comunidades - encerrada na estrutura de classes - continuava a realizar, apenas em outro cenário, a mesma medicina, sem crítica sobre seu agir social.

A formação da especialidade de MFC no Brasil, anterior à implementação da ESF, ajudou a compor um discurso sobre as práticas na APS. As primeiras residências datam de 1976, com o nome de Medicina Geral e Comunitária, denominação da especialidade até 2001, ano de alteração para MFC, mudança que refletia a construção de uma identidade que a distanciasse da Medicina Comunitária e da Medicina Preventiva ${ }^{7,8}$.

$\mathrm{O}$ afastamento em relação à Medicina Preventiva e à Comunitária demarcou também uma distinção da MFC em relação aos médicos sanitaristas e à Saúde Coletiva. Em paralelo à abertura de um amplo campo de práticas a partir da consolidação da ESF, a especialidade buscou afirmar-se enquanto campo autônomo, conectada ao ideário, práticas e conhecimentos de países com experiências mais consolidadas de atenção primária de primeiro nível como Canadá, Inglaterra e Espanha ${ }^{7,8,10}$.

Tais tensões expressaram-se ao interior da própria MFC, na defesa da especialidade no contexto da Reforma Sanitária e do SUS versus fortalecimento da especialidade autônoma na perspectiva liberal da profissão. A Sociedade Brasileira de Medicina Geral Comunitária, criada em 1981, precursora da Sociedade Brasileira de Medicina de Família e Comunidade (SBMFC), participou do movimento pela criação do SUS. Não obstante, em 2015, representantes da SBMFC, propuseram reassentar "as bases para um novo sanitarismo", a partir do acesso universal à saúde com financiamento público e prestação privada, contratualização de consultórios médicos, reversão do direito à saúde para direito a serviços de saúde custo efetivos, cadastro de pacientes e remuneração por misto de salário, capitação e desempenho ${ }^{11}$, em clara adaptação à prática liberal e ao mercado privado de saúde suplementar ${ }^{12}$. Em cenário atual, tais recomendações têm ecoado no direcionamento das políticas de atenção básica, sobretudo a partir da revisão da $\mathrm{PNAB}$ em $2017^{13}$, com possíveis implicações em componentes do modelo assistencial delineado a partir da ESF.

\section{Retrocessos do modelo assistencial da ESF nas políticas de atenção básica}

\section{Equipe multiprofissional}

A PNAB $2017^{13}$ permitiu estabelecer equipes de Saúde da Família (SF) com apenas um ACS e equipes AP (eAP) sem ACS. A possibilidade de ausência do ACS afeta um dos pilares do modelo assistencial que caracteriza a ESF em seu componente comunitário e de promoção da saúde, pautado pela concepção da determinação social do processo saúde-doença e da clínica amplia$\mathrm{da}^{14}$. Novas equipes com carga horária mínima do profissional, de dez horas semanais, restituem o emprego médico na atenção básica como um "bico" em vigor no período pré e nos primórdios do SUS. Tende ainda a fortalecer uma atuação profissional, sobretudo do médico, orientada à atenção curativa e de controle de riscos individuais $^{14}$. A nova política de financiamento da APS ${ }^{15}$ regulamenta as eAP que poderão receber incentivos financeiros equivalentes aos das equipes ESF.

O componente multiprofissional é enfraquecido também pela extinção do credenciamento e financiamento federal aos Núcleos de Apoio à Saúde da Família (NASF) ${ }^{15}$, sob a justificativa de maior autonomia do gestor municipal para a composição destas equipes. Incorporados à PNAB em $2011^{16}$, os NASF foram concebidos na ótica da interprofissionalidade, matriciamento, educação permanente, comunicação, planejamento conjunto, decisões, saberes e responsabilidade compartilhados, para uma maior resolutividade do cuidado, ações que provavelmente serão descontinuadas pela possibilidade concreta de demissão destes profissionais.

Com estas iniciativas, a própria ideia de multiprofissionalidade e interdisciplinaridade da ESF deixou de ser incentivada e em médio prazo tende a desaparecer, substituída por equipes cuja composição conste um profissional médico e um enfermeiro. 


\section{Prioridade ao cuidado individual \\ e à demanda espontânea}

O programa Saúde na $\mathrm{Hora}^{17}$, primeiro a ser lançado pelo MS no governo Bolsonaro, explicita a prioridade dada ao cuidado individual e ao atendimento à demanda espontânea. Nesta proposta, Unidades Básicas de Saúde (UBS) com no mínimo três equipes poderão oferecer atendimento de 60 horas semanais com flexibilização e redução de carga horária dos profissionais. Os incentivos para o Saúde na Hora apontam para a transformação das UBS, nos municípios de médio e grande porte, em unidades de pronto-atendimento ${ }^{18}$.

O acolhimento ao adoecimento agudo na APS certamente precisa ser garantido. Todavia, esta iniciativa tende a transformar a ESF em pronto -atendimento, descaracterizando seu processo de trabalho e organização das ações ${ }^{18}$. A abertura à contratação de plantonistas pode comprometer atributos da APS como a longitudinalidade e coordenação do cuidado. A proposta isolada, sem desenho de articulação com a rede de urgências, direciona a atenção às demandas agudas e manejo de doenças, com monitorização de casos graves à espera de transferência. Alerta-se também para a possibilidade de concentração das equipes nas áreas centrais dos municípios, perdendo-se a capilaridade da ESF nas comunidades.

\section{Gestão do trabalho médico para a APS}

O Programa Médicos pelo Brasil (PMB) ${ }^{19}$, alardeado como inovação, dá continuidade ao Programa Mais Médicos (PMM), em seu eixo de provisão de profissionais em zonas remotas e desfavorecidas. Abandona porém o componente de intervenção na graduação médica e ampliação das vagas de residência em MFC - padrão ouro para atuação em conformidade ao modelo assistencial da APS -, e o eixo voltado à melhoria da infraestrutura das UBS. Nesse sentido, apresenta escopo mais restrito que seu antecessor ${ }^{20}$.

A omissão a outros planos de formação para ESF, desde o nível de graduação até pós-graduação, indica que a reorientação para a prática médica na APS não será fomentada ${ }^{18,21}$. A manutenção dos atuais programas de Residência Médica em MFC será ainda mais difícil frente à proposição de formação prevista no PMB restrita a um curso de especialização cuja tutoria pode ser exercida por clínicos, e não médicos de família e comunidade $^{18}$. Equipara esta especialização à titulação por residência médica em MFC, encurtando pela metade o tempo exigido (quatros anos) para habilitação à prova de título da especialidade.
O PMB prevê a contratação de médicos com registro no país e, após bolsa por dois anos nos moldes do PMM, um contrato CLT intermediado por uma entidade privada sem fins de lucro, eufemisticamente denominada: Agência para o Desenvolvimento da APS (ADAPS) ${ }^{19}$. A ADAPS promove um deslocamento da gestão pública da APS para o âmbito privado ${ }^{18,21} \mathrm{em}$ um alinhamento às propostas de cobertura universal. $\mathrm{A}$ APS no SUS é reconhecida, até pelo Banco Mundial, como a área mais eficiente de todo o setor saúde brasileiro ${ }^{22}$, isso porque, a maior parte dos serviços de atenção básica é estatal, ofertada pela administração pública direta.

O conjunto de proposições abrigado no $\mathrm{PMB}^{19}$ se implementado, pode representar retorno ao modelo de assistência médica previdenciária, caminho para a privatização da APS, espaço até então, menos mercantilizado no SUS ${ }^{23}$.

\section{O escopo de ações/integralidade}

O escopo de práticas na APS certamente será afetado pelas ameaças à multiprofissionalidade das equipes, prioridade ao pronto atendimento e enfraquecimento do enfoque territorial comunitário. Os atributos derivados da APS são estruturantes de um modelo de APS abrangente e balizam como o cuidado em saúde deve ser desenvolvido ${ }^{18}$. Na política do governo Bolsonaro estes atributos vêm sendo desconsiderados nas diversas iniciativas e programas.

Em 2019, o MS divulgou uma proposição de "Carteira de Serviços da Atenção Primária à Saú$\mathrm{de}^{\prime 24}$ que, pela sua centralidade no cuidado médico individual, sofreu intensas críticas de pesquisadores e associações profissionais, incluindo o Conselho Nacional de Saúde ${ }^{25}$. A versão definitiva, pós consulta pública, corrigiu algumas das distorções iniciais, citando todos os atributos da APS e listando ações de vigilância, promoção da saúde e prevenção, conjugadas com amplo rol de cuidados clínicos individuais. Não obstante, o modelo assistencial expressado na carteira é de primeiro nível com ênfase no cuidado individual oportuno, denotando uma concepção restrita de APS, em detrimento de uma abordagem abrangente e da integração da APS à rede de serviços de saúde ${ }^{26}$. Este desequilíbrio pode ser ilustrado pela quase ausência de menção na carteira à ação dos ACS e à rede regionalizada. A versão final menciona o ACS somente uma vez, ao afirmar a necessidade de busca ativa de puérperas, e a integração à rede é mencionada apenas quando cita mecanismos de referência e contrarrefrência ${ }^{24}$. 
O novo modelo de financiamento da APS, ao substituir o Piso de Atenção Básica fixo e o variável para incentivo às equipes SF e ao NASF por um pagamento por capitação ponderada, calculado pelo número de pessoas cadastradas a equipes, pode ter efeitos drásticos no modelo assistencial da ESF.

O PAB fixo possibilita a implementação de ações previstas nos Planos Municipais de Saúde e mais afeitas às realidades locais, sem o engessamento comum nos repasses federais. No modelo até então vigente, as ações das equipes dirigemse a toda população do território, e, em centenas de municípios representam os únicos serviços de saúde disponíveis à população.

Mesmo que as diretrizes das atuais políticas de APS sinalizem a prioridade para o cuidado individual, as novas modalidades de financiamento poderão significar perdas expressivas para muitos municípios, já sobrecarregados. Estimativas do Cosems $\mathrm{SP}^{27}$ e do Cosems R ${ }^{28}$ de cálculo das transferências com base na capitação ponderada sinalizam enormes perdas. Nos 12 municípios da Baixada Fluminense, por exemplo, seria necessário cadastrar até maio de 2020 mais de 2 milhões de pessoas com uma perda mensal de seis milhões de reais, o que certamente resultará em desassistência à população ${ }^{28}$. Já o Cosems $\mathrm{SP}^{27}$ estima perda, em 2021, de 47\% dos recursos federais para APS nos municípios paulistas. Apesar das projeções ministeriais de algum aumento do financiamento para a APS em 2020, o que parece haver é uma realocação de recursos, com perdas evidentes para parte dos municípios, especialmente aqueles classificados como urbanos.

Outro componente do novo financiamento é o de desempenho que terá progressivamente um maior peso, conforme simulações preliminares

do MS. Mesmo sem representar recursos adicionais, como foi o caso do Programa Nacional de Melhoria do Acesso e da Qualidade da Atenção Básica, a nova proposta de desempenho também terá grande ênfase no redirecionamento das práticas.

Além das possíveis perdas de recursos financeiros, a opção política parece ser pela focalização e seletividade. O novo financiamento prejudica a responsabilidade constitucional do SUS sobre a segurança sanitária e prevenção de riscos, dado que o cuidado se restringirá ao público "cadastrado", comprometendo as ações coletivas de promoção da saúde. Considerar apenas a população cadastrada, na prática, significa romper com a universalidade e equidade do SUS.

\section{Considerações finais}

Aliar boa prática clínica, compromisso com a prevenção de doenças e a promoção da saúde, amplo acesso aos serviços, cuidado multiprofissional interdisciplinar, vinculação aos territórios, participação da comunidade e incidência sobre determinantes sociais são desafios que sempre estiveram presentes na efetivação de um novo modelo assistencial no SUS, na perspectiva da saúde como direito universal.

Romper com a universalidade do SUS, como pretendido e implementado pelo atual governo com base em um discurso supostamente pró-equidade é uma falácia. Trata-se de um processo de "neoseletividade" caracterizado pela oferta de ações de saúde com financiamento público aos estratos populacionais extremamente pobres, por prestadores privados ou públicos, apartada de uma perspectiva de redes e regiões de saúde, em consonância com as políticas restritivas de ajuste fiscal e de redução da intervenção estatal. O conjunto de reformas das políticas sociais, incluindo-se as do setor saúde, empreendidas de forma voraz e açodada pelo governo Bolsonaro acentua e cristaliza iniquidades, e fortalece a mercantilização também na prestação de serviços de APS. 


\section{Colaboradores}

L Giovanella, CM Franco e PF Almeida participaram da concepção, análise e redação do texto.

\section{Referências}

1. Paim JS. Modelos de atenção à saúde no Brasil. In: Giovanella L, Escorel S, Lobato LVV, Noronha JC, Carvalho AI, organizadores. Políticas e Sistema de Saúde no Brasil. Rio de Janeiro: Ed. Fiocruz; 2012. p. 459-491.

2. Teixeira CF. Promoção e vigilância da saúde no contexto da regionalização da assistência à saúde no SUS. Cad Saude Publica 2002; 18(Supl.):S153-S162.

3. Viana AL, Dal Poz MR. A Reforma do Sistema de Saúde no Brasil e o Programa de Saúde da Família. PHYSIS 2005; 15(Supl.):225-264.

4. Giovanella L, Mendonça MH. Atenção primária à saúde. In: Giovanella L, Escorel S, Lobato LVV, Noronha JC, Carvalho AI, organizadores. Políticas e Sistema de Saúde no Brasil. Rio de Janeiro: Ed. Fiocruz; 2012. p. 493-545

5. Starfield B. Atenção Primária: equilíbrio entre necessidades de saúde, serviços e tecnologia. Brasília: UNESCO, MS; 2002.

6. Brasil. Ministério da Saúde (MS). Portaria no 648, de 28 de março de 2006. Dispõe sobre a revisão de diretrizes e normas para a organização da Atenção Básica para o Programa da Saúde da Família (PSF) e o Programa de Agentes Comunitários de Saúde (PACS). Diário Oficial da União 2006; 28 mar.

7. Andrade HS, Alves MGM, Carvalho SR, Silva Jr AG. A formação discursiva da Medicina de Família e Comunidade no Brasil. PHYSIS 2018; 28(3):e280311.

8. Falk JW. A medicina de família e comunidade e sua entidade nacional: histórico e perspectivas. Rev Bras Med Fam Comunidade 2004; 1(1):5-10.

9. Donnangelo C. Saúde e Sociedade. São Paulo: Hucitec; 1979.

10. Trindade TG, Batista SR. Medicina de Família e Comunidade: agora mais do que nunca! Cien Saude Colet 2016; 21(9):2667-2669.

11. Gusso GDF, Knupp D, Trindade TG, Lermen Junior N, Poli Neto P. Bases para um Novo Sanitarismo. Rev Bras Med Fam Comunidade 2015; 10(36):1-10.

12. Machado HSV, Melo EA, Paula LGN. Medicina de Família e Comunidade na saúde suplementar do Brasil: implicações para o Sistema Único de Saúde e para os médicos. Cad Saude Publica 2019; 35(11):e00068419.

13. Brasil. Ministério da Saúde (MS). Portaria no 2.436 de 21 de setembro de 2017. Aprova a Política Nacional de Atenção Básica, estabelecendo a revisão de diretrizes para a organização da Atenção Básica, no âmbito do Sistema Único de Saúde (SUS). Diário Oficial da União; 2017.

14. Morosini MVGC, Fonseca AF, Lima LD. Política Nacional de Atenção Básica 2017: retrocessos e riscos para o Sistema Único de Saúde. Saude Debate 2018; 42(116):11-24. 
15. Brasil. Ministério da Saúde (MS). Portaria no 2.979, de 12 de novembro de 2019. Institui o Programa Previne Brasil. Diário Oficial da União 2019; 13 nov.

16. Brasil. Ministério da Saúde (MS). Portaria no 2.488 , de 21 de outubro de 2011. Aprova a Política Nacional de Atenção Básica, estabelecendo a revisão de diretrizes e normas para a organização da Atenção Básica, para a Estratégia Saúde da Família (ESF) e o Programa de Agentes Comunitários de Saúde (PACS). Diário Oficial da União 2011; 22 out.

17. Brasil. Ministério da Saúde (MS). Portaria no 930, de 15 de maio de 2019. Institui o Programa "Saúde na Hora", que dispõe sobre o horário estendido de funcionamento das Unidades de Saúde da Família. Diário Oficial da União 2019; 17 mai.

18. Anderson MIP. Médicos pelo Brasil e as políticas de saúde para a Estratégia Saúde da Família de 1994 a 2019: caminhos e descaminhos da Atenção Primária no Brasil. Rev Bras Med Fam Comunidade 2019; 14(41):2180.

19. Brasil. Lei no 13.958, de 18 de dezembro de 2019. Institui o Programa Médicos pelo Brasil. Diário Oficial da União 2019; 19 dez.

20. Melo Neto AJ, Barreto DS. Programa Médicos pelo Brasil: inovação ou continuidade? Rev Bras Med Fam Comunidade 2019; 14(41):2162.

21. Giovanella L, Bousquat A, Almeida PF, Melo EA, Medina MG, Aquino R, Mendonça MHM. Médicos pelo Brasil: caminho para a privatização da atenção primária à saúde no Sistema Único de Saúde? Cad Saude Publica 2019; 35(10):e00178619.

22. Banco Mundial. Um Ajuste Justo: Análise da eficiência e equidade do gastopúblico no Brasil.Brasil revisão das despesas públicas. Volume I: Síntese [Internet]. 2017 [acessado 2020 Fev 07]. Disponível em http://documents. worldbank.org/curated/en/884871511196609355/ pdf/121480-REVISED-PORTUGUESE-Brazil-Public-Expenditure-Review-Overview-Portuguese-Final-revised.pdf

23. Viana ALd'A, Fausto MRC. Atenção básica e proteção social: universalismo $\mathrm{x}$ focalismo e espaço não mercantil da assistência. In: Viana ALd'A, Elias PEM, Ibañez $\mathrm{N}$, organizadores. Proteção social: dilemas e desafios. São Paulo: Editora Hucitec; 2005. p. 150-168.

24. Ministério da Saúde (MS). Secretaria de Atenção Primária à Saúde (SAPS). Carteira de Serviços da Atenção Primária à Saúde (CaSAPS) [Internet]. Brasília: SAPS; 2019 [acessado 2020 Fev 08]. Disponível em: http://189.28.128.100/dab/docs/portaldab/documentos/casaps_versao_profissionais_saude gestores_completa.pdf

25. Conselho Nacional de Saúde (CNS). Recomendação $\mathrm{n}^{\circ}$ 035, de 23 de agosto de 2019 [Internet]. 2019 [acessado $2020 \mathrm{Fev}$ 05]. Disponível em: https:// drive.google.com/file/d/1qpYmzHgGQKG7aqmlM_ GM3u2y8d3s3ZFx/view
26. Rede de Pesquisa em APS da Abrasco (Rede APS). Contribuição dos pesquisadores da Rede APS ao debate sobre as recentes mudanças na política de atenção primária propostas pelo MS [Internet]. João Pessoa: Rede APS; 2019. [acessado $2020 \mathrm{Fev} 11$ ]. Disponível em: https:// redeaps.org.br/wp-content/uploads/2019/10/Contribuicoesdospesquisaadores_REDEAPS.pdf

27. Conselho de Secretarias Municipais de Saúde RJ (Cosems RJ). Nota técnica Cosems RJ no 02/2019. Análise do impacto da modificação dos critérios de financiamento da Atenção Básica nos municípios do Estado do Rio de Janeiro para os anos de 2020 e 2021 [Internet]. 2019 [acessado $2020 \mathrm{Fev}$ 10]. Disponível em: http://www.cosemsri.org.br/wp-content/uploads /2019/12/Estudo-do-Impacto-do-Atual-Financiamento -da-APS-nos-Municipios-do-ERJ_final_16dez 2019.-1.pdf

28. Conselho de Secretarias Municipais de Saúde SP (Cosems SP). Nota Técnica Cosems SP no 1/2019. Novo modelo de financiamento da Atenção Primária em Saúde [Internet]. 2019 [acessado 2020 Fev 10]. Disponível em: http://www.cosemssp.org.br/noticias/ nota-tecnica-cosems-sp-novo-modelo-de-financiamento-da-atencao-primaria-em-saude/

Artigo apresentado em 05/12/2019

Aprovado em 15/01/2020

Versão final apresentada em 17/01/2020 
